\title{
Linear and Nonlinear Boundary Conditions for Wave Propagation Problems
}

\author{
Jan Nordström
}

\section{Linköping University Post Print}

N.B.: When citing this work, cite the original article.

Original Publication:

Jan Nordström, Linear and Nonlinear Boundary Conditions for Wave Propagation Problems, in Recent Developments in the Numerics of Nonlinear Hyperbolic Conservation Laws: Notes on Numerical Fluid Mechanics and Multidisciplinary Design, eds Rainer Ansorge , Hester Bijl, Andreas Meister and Thomas Sonar, Springer, 2013, 283-299, ISBN 978-3-642-332203.

http://dx.doi.org/10.1007/978-3-642-33221-0_17

Copyright: Springer

Postprint available at: Linköping University Electronic Press

http://urn.kb.se/resolve?urn=urn:nbn:se:liu:diva-84545 


\title{
Linear and Nonlinear Boundary Conditions for Wave Propagation Problems
}

Jan Nordström

\begin{abstract}
We discuss linear and nonlinear boundary conditions for wave propagation problems. The concepts of well-posedness and stability are discussed by considering a specific example of a boundary condition occurring in the modeling of earthquakes. That boundary condition can be formulated in a linear and nonlinear way and implemented in a characteristic and non-characteristic way. These differences are discussed and the implications and difficulties are pointed out. Numerical simulations that illustrate the theoretical discussion are presented together with an application that show that the methodology can be used for practical problems.
\end{abstract}

\section{Introduction}

The principles for construction stable and convergent high order finite difference schemes for linear and nonlinear boundary conditions are discussed in the context of wave propagation problems in earthquake simulations.

\subsection{Recipe for Constructing a Scheme}

The first requirement for obtaining a reliable solution is well-posedness, see [6],[16]. A well posed problem is bounded by the data of the problem and has a unique solution. Uniqueness for linear problems follows more or less directly from the energy estimate. This is however not the case for nonlinear problems and we will investigate that in detail below. Existence is motivated by using a minimal number of boundary conditions. In the rest of the paper we assume that existence is not a

Jan Nordström

Division of Computational Mathematics, Department of Mathematics, Linköping University, 581

83 Linköping Sweden e-mail: jan.nordstrom@liu.se 
problem and will not discuss it further. The crucial point in obtaining well-posedness is the boundary conditions. These will be chosen such that an energy estimate is obtained with a minimal number of conditions.

Once we have a well-posed problem, it is meaningful to construct a numerical approximation. We will use high order finite differences on Summation-By-Parts (SBP) form and impose the boundary conditions weakly using penalty terms. More details on this productive and well tested technique is given below. For a read-up, see [3],[11],[13],[14],[20],[19],[21],[15], [2],[5]. A recipe for constructing a stable and convergent scheme when using the SBP-SAT technique is to choose the so called penalty parameters such that an energy-estimate is obtained.

For linear problems, the recipe outlined above guarantees that the scheme converges to a reliable solution as the mesh size goes to zero. However, as we shall see below, this is not always the case for nonlinear boundary conditions. The difference in analysis due to the linear and nonlinear version of the boundary condition/friction law is the main topic of this paper.

\subsection{Modeling Related to Earthquake Simulations}

The material is modeled as linear elastic with frictional sliding occurring on thin internal interfaces. The internal interfaces, or faults, are governed by highly nonlinear friction laws. The friction laws relate the slip velocities to the tractions acting on the fault. They constitute a set of nonlinear boundary/interface conditions. The elastic wave equations govern the wave propagation between the faults. For a read-up on these problems see [8],[9].

A simplified but realistic model of problems of this sort is given by

$$
u_{t}=A u_{y}, \quad y \geq 0, \quad u=\left(\begin{array}{c}
v \\
\sigma
\end{array}\right), \quad A=\left(\begin{array}{ll}
0 & 1 \\
1 & 0
\end{array}\right), \quad u(y, 0)=f(y) .
$$

Note that we have both ingoing and outgoing waves at the boundary $y=0$ where the fault is located. At $y=0$ we have either a linear friction law $\sigma=\lambda \nu$ with $\lambda$ being a constant or, a highly nonlinear friction law of the general form $\sigma=F(v)$. The relation between the velocity and stress is visualized in Figure 1.

We conclude this section by making the assumption that all solutions decay as $y$ increases i.e $\lim _{y \rightarrow \infty} u=0$. This assumption simplifies the analysis and enables us to focus on the interesting boundary with the friction law. In the rest of this paper, all boundary terms are evaluated at $y=0$. The boundary terms for large $y$ are neglected. 


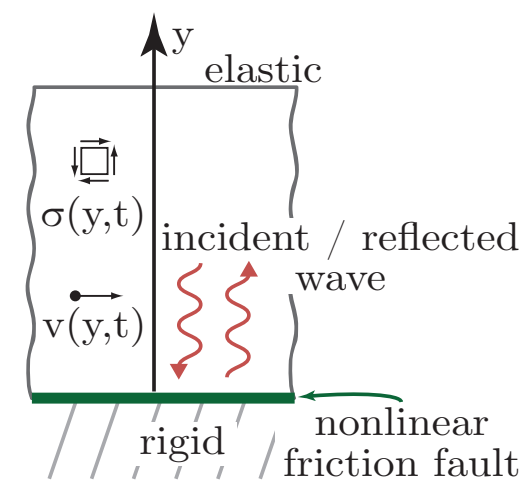

Fig. 1 A schematic of a fault with one-sided friction laws. The lower part of the fault is rigid.

\section{Analysis}

Below we outline the standard recipe for constructing a stable scheme for a linear problem. The nonlinear boundary condition will force us to slightly modify that.

\subsection{Well-posedness}

We will consider two different formulations with slightly different characters.

\subsubsection{Non Characteristic Formulation}

The energy-method applied to (1 ) yields:

$$
2 \int_{0}^{\infty} u^{T} u_{t} d y=\|u\|_{t}^{2}=-2 v \sigma .
$$

To obtain a bounded solution $\|u\|^{2} \leq\|f\|^{2}$, the linear and nonlinear friction laws must obey

$$
\lambda \geq 0, \quad v F(v) \geq 0
$$

respectively.

Next we consider uniqueness and start with the linear case. Consider the difference problem for $\Delta u=u_{1}-u_{2}$,

$$
\Delta u_{t}=A \Delta u_{y}, \quad y \geq 0, \quad \Delta u=\left(\begin{array}{c}
\Delta v \\
\Delta \sigma
\end{array}\right), \quad \Delta u(y, 0)=0
$$

and the boundary condition $\Delta \sigma=\sigma_{1}-\sigma_{2}=\lambda \Delta \nu$. The energy-method yields: 


$$
\|\Delta u\|_{t}^{2}=-2 \Delta v \Delta \sigma=-\lambda \Delta v^{2}
$$

and clearly the first condition in (2) that guarantees a bounded energy also guarantees uniqueness (since we obtain $\|\Delta u\|^{2} \leq 0$ by integrating (4)). We summarize the result in the following Theorem.

Theorem 1. The problem (1) with the linear boundary condition $\sigma=\lambda v$ is well posed if

$$
\lambda \geq 0 .
$$

In the nonlinear case we have $\Delta \sigma=\sigma_{1}-\sigma_{2}=F\left(v_{1}\right)-F\left(v_{2}\right)$. The energymethod applied to the difference equation (3) yields:

$$
\|\Delta u\|_{t}^{2}=-2 \Delta v \Delta \sigma=-F^{\prime}(v) \Delta v^{2}
$$

where the intermediate value theorem has been used and $v \in\left(v_{1}, v_{2}\right)$. Note that an additional condition, namely $F^{\prime}(v) \geq 0$ must be added on to the second condition in (2) which lead to an energy estimate. We summarize the result in the following Theorem.

Theorem 2. The problem (1) with the nonlinear boundary condition $\sigma=F(v)$ is well posed if

$$
v F(v) \geq 0 \quad \text { and } \quad F^{\prime}(v) \geq 0
$$

\subsubsection{Characteristic Formulation}

The characteristic formulation is obtained by diagonalizing (1). The result if

$$
w_{t}=\Lambda w_{y}, \quad w=\left(\begin{array}{c}
w^{+} \\
w^{-}
\end{array}\right), \quad \Lambda=\left(\begin{array}{cc}
-1 & 0 \\
0 & +1
\end{array}\right), \quad w(y, 0)=h(y) .
$$

The relation between the characteristic variables and the standard variables are

$$
\sigma=\left(w^{-}+w^{+}\right) / 2, \quad v=\left(w^{-}-w^{+}\right) / 2 .
$$

The friction laws can now be formulated as reflection relations. In the linear case we have

$$
w^{+}=R w^{-}, \quad R=(\lambda-1) /(\lambda+1) .
$$

In the nonlinear case we need to be more careful. The nonlinear friction law implies $\sigma-v=F(v)-v$ which by the use of (9) lead to

$$
w^{+}=F(v)-v=H(v)=H\left(\left(w^{-}-w^{+}\right) / 2\right) .
$$

According to the implicit function theorem, (11) uniquely determines the ingoing characteristic variable $w^{+}$in terms of the outgoing $w^{-}$if

$$
\frac{\partial}{\partial w^{+}}\left(w^{+}-H\left(\left(w^{-}-w^{+}\right) / 2\right)\right) \neq 0 .
$$


Consequently, the condition for a unique solution becomes $H^{\prime}(v) \neq-2$ or

$$
F^{\prime}(v) \neq-1 \text {. }
$$

The nonlinear friction law on reflection form can be derived as follows. We have

$$
w^{+}=\sigma-v=F(v)-v=\frac{F(v)-v}{\sigma+v}(\sigma+v)=\left(\frac{F(v)-v}{F(v)+v}\right) w^{-} .
$$

We summarize the result as

$$
\left.w^{+}=R w^{-}, \quad R=(F(v)-v) /(F(v)+v)\right)=R\left(\left(w^{-}-w^{+}\right) / 2\right) .
$$

To prove well-posedness in the nonlinear characteristic case we need the weighted norm

$$
\int_{0}^{\infty} w^{T} B w d y=\|w\|_{B}^{2} \quad B=\left(\begin{array}{ll}
\delta & 0 \\
0 & 1
\end{array}\right), \quad \delta>0 .
$$

The energy-method using the new norm and the reflection condition yields

$$
\left(\|w\|_{B}^{2}\right)_{t}=-\left(w^{-}\right)^{2}\left(1-\delta R^{2}\right) .
$$

In the linear case (10) we always have $|R|<1$ since $\lambda>0$ and we immediately have an estimate even for a standard norm with $\delta=1$.

In the nonlinear case, it is not that simple. However, If $|R| \leq C<\infty$ then $0<\delta<$ $1 / C^{2}$ guarantees the estimate. We need to show that $|R|$ is bounded. The extreme value of $R=(F(v)-v) /(F(v)+v))$ is given by

$$
R^{\prime}(v)=2\left(F^{\prime}(v) v-F(v)\right) /(F(v)+v)^{2}=0
$$

which is zero for $F(v)=F^{\prime}(v) v$. The extreme value is $R=\left(F^{\prime}(v)-1\right) /\left(F^{\prime}(v)+1\right)$, and hence condition (12) also leads to an energy-estimate. Note that also the first condition in (7) is required to keep $|R|$ bounded.

Next we turn to the question of uniqueness. The energy-method on the difference problem using (14) yields

$$
\left(\|d w\|_{B}^{2}\right)_{t}=-\left(\left(d w^{-}\right)^{2}-\delta\left(d w^{+}\right)^{2}\right) .
$$

where $d w^{+/-}=w_{1}^{+/-}-w_{2}^{+/-}$and $d w=\left(d w^{+}, d w^{-}\right)^{T}$. In the linear case (10) leads directly to $d w^{+}=R d w^{-}$and uniqueness after integration in time. We summarize the result as

Theorem 3. The problem (8) with the linear characteristic boundary condition (10) is well posed.

In the nonlinear case we need to derive the relation $d w^{+}=d R d w^{-}$. By the intermediate value theorem we get

$$
\sigma_{1}-\sigma_{2}=F\left(v_{1}\right)-F\left(v_{2}\right)=F^{\prime}(v)\left(v_{1}-v_{2}\right), \quad v \in\left(v_{1}, v_{2}\right) .
$$


Reformulating in terms of difference variables leads to

$$
d R=\left(F^{\prime}(v)-1\right) /\left(F^{\prime}(v)+1\right),
$$

and consequently, by the same arguments as in the energy-estimate, $F^{\prime}(v) \neq-1$ leads to uniqueness. We summarize the result as

Theorem 4. The problem (8) with the nonlinear characteristic boundary condition (13) is well posed if

$$
v F(v) \geq 0 \quad \text { and } \quad F^{\prime}(v) \neq-1 .
$$

The condition (16) is less restrictive than (7) (allows for more general types of friction laws). We also see that the nonlinear case is more complex than the linear case where condition (5) suffice for all formulations.

\subsection{Stability}

We will use high order finite difference techniques on Summation-By-Parts (SBP) form and impose the boundary conditions (the friction laws) weakly using the Simultaneous Approximation Term (SAT) technique.

\subsubsection{SBP Operators and Weak Non Characteristic Boundary Conditions}

The semi-discrete formulation of (1) with the weakly enforced boundary condition is:

$$
\mathbf{U}_{t}=\left(P^{-1} Q \otimes A\right) \mathbf{U}+P^{-1} e_{0} \otimes \Sigma \mathbf{B}\left(\mathbf{U}_{0}\right)
$$

where $e_{0}=(1,0, \cdots, 0)^{T}, \otimes$ is the Kronecker product, $\Sigma=\left(\Sigma_{1}, \Sigma_{2}\right)$ is the penalty matrix, $\mathbf{U}=\left(\mathbf{U}_{0}, \mathbf{U}_{1}, \cdots, \mathbf{U}_{N}\right)^{T}, \mathbf{U}_{i}=\left(v_{i}, \sigma_{i}\right)^{T}$ and

$$
\mathbf{B}_{s}\left(\mathbf{U}_{0}\right)=(1,1)^{T}\left[\sigma_{0}-F\left(v_{0}\right)\right] .
$$

We augment (17),(18) with the initial condition $\mathbf{U}(\mathbf{0})=\mathbf{f}$.

Note that we have expressed both the linear and nonlinear standard boundary condition in the same functional form $\left(\sigma_{0}=F\left(v_{0}\right)\right)$. We have used a summationby-parts (SBP) difference operator $P^{-1} Q$ (see [10],[18]) and imposed the boundary conditions weakly using the Simultaneous Approximation Term (SAT) technique [3]. The SBP difference operators satisfy the relations

$$
P=P^{T}>0, \quad Q+Q^{T}=E_{N}-E_{0}=\operatorname{diag}(-1,0, \ldots 0,1),
$$

and hence they mimic integration by parts perfectly. More details on the weak imposition of boundary and interface conditions using the SAT technique will be given below. For a read-up on this technique see [3],[11],[13],[14],[20],[19],[21],[15].

By multiplying (17) from the left with $\mathbf{U}^{T}(P \otimes I)$ we obtain 


$$
\frac{d}{d t}\|\mathbf{U}\|_{h}^{2}=-2 v_{0} \sigma_{0}+2 \mathbf{U}_{0}^{T} \Sigma \mathbf{B}\left(\mathbf{U}_{0}\right) .
$$

The choice $\Sigma_{1}=1$ and $\Sigma_{2}=0$ leads to

$$
\frac{d}{d t}\|\mathbf{U}\|_{h}^{2}=-2 v_{0} F\left(v_{0}\right)
$$

which is completely similar to the continuous estimates in both the linear and nonlinear case. We summarize the result below.

Theorem 5. The approximation (17) of the problem (1) is stable for both the linear (5) and nonlinear (7) boundary condition if the penalty coefficients $\Sigma_{1}=1$ and $\Sigma_{2}=0$ are used.

Note that the conditions (5) and (7) that lead to well-posedness in the continuous case, are necessary for stability.

\subsubsection{SBP Operators and Weak Characteristic Boundary Conditions}

The semi-discrete formulation of (8) with the weakly enforced characteristic boundary conditions is:

$$
\mathbf{W}_{t}=\left(P^{-1} Q \otimes \Lambda\right) \mathbf{W}+P^{-1} e_{0} \otimes \Sigma \mathbf{B}\left(\mathbf{W}_{0}\right)
$$

where $\mathbf{W}_{i}=\left(w_{i}^{+}, w_{i}^{-}\right)^{T}$ and

$$
\mathbf{B}_{c}\left(\mathbf{U}_{0}\right)=(1,1)^{T}\left[w_{0}^{+}-R w_{0}^{-}\right] .
$$

We augment (21),(22) with the initial condition $\mathbf{W}(\mathbf{0})=\mathbf{h}$. Note that we have expressed both the linear and nonlinear characteristic boundary condition in the same functional form $\left(w_{0}^{+}=R w_{0}^{-}\right)$.

By multiplying (21) from the left with $\mathbf{W}^{T}(P \otimes B)$ where $B=\operatorname{diag}(\delta, 1)$ gives us the weighted norm required above for well-posedness. We obtain

$$
\frac{d}{d t}\|\mathbf{W}(t)\|_{h}^{2}=\delta\left(w_{0}^{+}\right)^{2}-\left(w_{0}^{-}\right)^{2}+2 \mathbf{W}_{0}^{T} \Sigma \mathbf{B}_{c}\left(\mathbf{W}_{0}\right) .
$$

Th choice $\Sigma_{1}=-\delta$ and $\Sigma_{2}=0$ leads to the estimate

$$
\frac{d}{d t}\|\mathbf{W}(t)\|_{h}^{2}=-\left(w_{0}^{-}\right)^{2}\left(1-\delta R^{2}\right)-\delta\left(w_{0}^{+}-R w_{0}^{-}\right)^{2},
$$

which is similar to the continuous one both in the linear and nonlinear case. Note that a small damping term proportional to the deviation in the boundary conditions is added on. We summarize the result in the following theorem. 
Theorem 6. The approximation (21) of the problem (8) is stable for both the linear (10) and nonlinear (13) boundary condition if the penalty coefficients $\Sigma_{1}=-\delta$ and $\Sigma_{2}=0$ are used .

Note that the condition (5) and (16) that lead to well-posedness in the continuous case, are necessary for stability. Note also that in the linear case we always have $|R|<1$ since $\lambda>0$ and just as in the continuous case, we immediately have an estimate even for a standard norm with $\delta=1$.

\subsection{Convergence for Finite Time}

We will derive the error equation and investigate under which requirement the numerical solution converge to the analytic solution.

\subsubsection{Weak Non Characteristic Boundary Conditions}

By inserting the analytical solution $\overline{\mathbf{U}}$ (projected onto the mesh) in (17) and subtracting (17) we obtain the error equation

$$
\mathbf{E}_{t}=\left(P^{-1} Q \otimes A\right) \mathbf{E}+P^{-1} e_{0} \otimes \Sigma \mathbf{B}\left(\mathbf{E}_{0}\right)+\mathbf{T}_{\mathbf{e}}
$$

where $\mathbf{E}=\overline{\mathbf{U}}-\mathbf{U}, \mathbf{E}=\left(\mathbf{E}_{0}, \mathbf{E}_{1}, \cdots, \mathbf{E}_{N}\right)^{T}, \mathbf{E}_{i}=\left(\Delta v_{i}, \Delta \sigma_{i}\right)^{T}$ is the error in the numerical solution, $\mathbf{T}_{\mathbf{e}}=\mathbf{O}\left(\Delta x^{p}\right)$ is the truncation error and

$$
\mathbf{B}_{s}\left(\mathbf{E}_{0}\right)=(1,1)^{T}\left[\Delta \sigma_{0}-\left(F\left(\bar{v}_{0}\right)-F\left(v_{0}\right)\right)\right] .
$$

The initial data is zero (we initiate the numerical solution with the exact initial data projected onto the grid), i.e. $\mathbf{E}(\mathbf{0})=0$.

In this paper we assume that the truncation error $\mathbf{T}_{\mathbf{e}}=\mathbf{O}\left(\Delta x^{p}\right)$ is uniform in accuracy. In reality, the accuracy close to the boundaries are lower, see [18]. This is especially true for diagonal norm $\mathbf{P}$ which one needs in many cases for stability reasons, see for example [14],[17]

By multiplying (24) from the left with $\mathbf{E}^{T}(P \otimes I)$ we obtain

$$
\frac{d}{d t}\|\mathbf{E}\|_{h}^{2}=-2 \Delta v_{0}\left(F\left(\bar{v}_{0}\right)-F\left(v_{0}\right)\right)+2 \mathbf{E}^{T}(P \otimes I) \mathbf{T}_{\mathbf{e}},
$$

where $\Sigma_{1}=1$ and $\Sigma_{2}=0$ have been used. In the linear case the first term is negative by the fact that condition (5) holds. In the nonlinear case, the intermediate value theorem in combination with the second condition in (7) leads to the same result.

The negative contribution of the first term in (26) and the standard inequality

$$
2(u, v) \leq \eta\|u\|^{2}+(1 / \eta)\|v\|^{2}
$$


leads to

$$
\frac{d}{d t}\|\mathbf{E}\|_{h}^{2} \leq \eta\|\mathbf{E}\|_{h}^{2}+(1 / \eta)\left\|\mathbf{T}_{\mathbf{e}}\right\|_{h}^{2} .
$$

Time integration of (28) leads to the final accuracy result

$$
\|\mathbf{E}(T)\|_{h}^{2} \leq \frac{e^{\eta T}}{\eta} \int_{0}^{T} e^{-\eta t}\left\|\mathbf{T}_{\mathbf{e}}\right\|_{h}^{2} d t=\mathbf{O}\left(\Delta x^{2 p}\right),
$$

which we summarize below.

Theorem 7. The solution of the approximation (17) converges to the solution of the problem (1) with linear (5) and nonlinear (7) boundary condition if the penalty coefficients $\Sigma_{1}=1$ and $\Sigma_{2}=0$ are used.

\subsubsection{Weak Characteristic Boundary Conditions}

We proceed in the same way as in the previous section and insert the analytical solution $\overline{\mathbf{W}}$ projected onto the mesh in (21) and subtract (21) to obtain the error equation

$$
\mathbf{E}_{t}=\left(P^{-1} Q \otimes \Lambda\right) \mathbf{E}+P^{-1} e_{0} \otimes \Sigma \mathbf{B}\left(\mathbf{E}_{0}\right)+\mathbf{T}_{\mathbf{e}}
$$

where $\mathbf{E}_{i}=\overline{\mathbf{W}}_{i}-\mathbf{W}_{i}=\left(\Delta w_{i}^{+}, \Delta w_{i}^{-}\right)^{T}, \mathbf{T}_{\mathbf{e}}=\mathbf{O}\left(\Delta x^{p}\right)$ is the truncation error and

$$
\mathbf{B}_{c}\left(\mathbf{E}_{0}\right)=(1,1)^{T}\left[\Delta w_{0}^{+}-\Delta\left(R w_{0}^{-}\right)\right] .
$$

Precisely as in the previous section $\mathbf{E}(\mathbf{0})=0$. Note that we have expressed both the linear and nonlinear characteristic boundary condition in the same functional form $\left(\Delta w_{0}^{+}=\Delta\left(R w_{0}^{-}\right)\right)$.

By multiplying (30) from the left with $\mathbf{E}^{T}(P \otimes B)$ (using the weighted norm) we obtain

$$
\frac{d}{d t}\|\mathbf{E}\|_{h}^{2}=\delta\left(\Delta w_{0}^{+}\right)^{2}-\left(\Delta w_{0}^{-}\right)^{2}+2 \mathbf{E}_{0}^{T} \Sigma \mathbf{B}_{c}\left(\mathbf{E}_{0}\right)+2 \mathbf{E}^{T}(P \otimes B) \mathbf{T}_{\mathbf{e}} .
$$

The relations (11) and (9) together with the intermediate value theorem leads to

$$
\Delta w_{0}^{+}-\Delta\left(R w_{0}^{-}\right)=\Delta w_{0}^{+}-H^{\prime}(\hat{v}) \Delta v=\left(\frac{F^{\prime}+1}{2}\right) \Delta w_{0}^{+}-\left(\frac{F^{\prime}-1}{2}\right) \Delta w_{0}^{-},
$$

where $\hat{v} \in(\bar{v}, v)$. By inserting $\Sigma_{1}=-\delta, \Sigma_{2}=0$ and (33) in (32) we get

$$
\frac{d}{d t}\|\mathbf{E}\|_{h}^{2}=\mathbf{E}_{0}^{T}\left(\begin{array}{cc}
-\delta F^{\prime} & \delta\left(F^{\prime}-1\right) / 2 \\
\delta\left(F^{\prime}-1\right) / 2 & -1
\end{array}\right) \mathbf{E}_{0}+2 \mathbf{E}^{T}(P \otimes B) \mathbf{T}_{\mathbf{e}} .
$$

By computing the eigenvalues of the boundary matrix and demanding them to be negative, we find that we must satisfy the second condition in (7). 
Consequently, the same conditions an in the non-characteristic case are required also for the characteristic case although it seemed less restrictive for a while. If the second condition in (7) holds, we can use (27) and (28) and obtain an estimate like (29) also in the characteristic case. This proves that the solution converges to the correct solution using both types of boundary conditions. We summarize that in the following Theorem.

Theorem 8. The solution of the approximation (21) converges to the solution of the problem (8) with linear (10) and nonlinear (13) boundary condition if the penalty coefficients $\Sigma_{1}=-\delta$ and $\Sigma_{2}=0$ are used. In the nonlinear case, also the condition (7) must hold.

Note that condition (16) does not suffice for convergence.

\subsection{An Error Bound in Time}

We will show that under certain reasonable assumptions, the error growth in time is bounded even for long times, see [1] and in particular [12]. Both the equations (26) and (34) can be written

$$
\frac{d}{d t}\|\mathbf{E}\|_{h}^{2} \leq-2 C_{0}\left|\mathbf{E}_{0}\right|^{2}+2\|\mathbf{E}\|\left\|\mathbf{T}_{\mathbf{e}}\right\|,
$$

where $C_{0}$ is an appropriate non-zero constant. By expanding the left-hand side as $\frac{d}{d t}\|\mathbf{E}\|_{h}^{2}=2\|\mathbf{E}\|_{h} \frac{d}{d t}\|\mathbf{E}\|_{h}$ we get

$$
\frac{d}{d t}\|\mathbf{E}\|_{h} \leq-\eta(t)\|\mathbf{E}\|_{h}+\left\|\mathbf{T}_{\mathbf{e}}\right\|
$$

where $\eta(t)=C_{0}\left|\mathbf{E}_{0}\right|^{2} /\|\mathbf{E}\|_{h}^{2}$.

For the sake of argument, we assume that $\eta(t)=\eta=$ const. independent of time. In that case we can integrate (36) and obtain $\|\mathbf{E}(T)\|_{h} \leq e^{-\eta T} \int_{0}^{T} e^{\eta t}\left\|\mathbf{T}_{\mathbf{e}}(t)\right\|_{h} d t$. The estimate $\left\|\mathbf{T}_{\mathbf{e}}(t)\right\|_{h} \leq \max _{0 \leq t \leq T}\left\|\mathbf{T}_{\mathbf{e}}(t)\right\|_{h}=\left(\left\|\mathbf{T}_{\mathbf{e}}\right\|_{h}\right)_{\max }$ leads to the final error bound

$$
\|\mathbf{E}(T)\|_{h} \leq\left(\left\|\mathbf{T}_{\mathbf{e}}\right\|_{h}\right)_{\max } \frac{\left(1-e^{-\eta T}\right)}{\eta} .
$$

In the case of a time-dependent $\eta(t)$ not much is changed as long as $\eta(t)$ is nonnegative and monotonically increasing. The conclusion (37) still holds, see [12]. In conclusion we have that both the characteristic and non-characteristic boundary conditions imposed weakly lead to an error bound in time. 


\section{Numerical Results}

The results presented here are given in [8],[9],[7],[4]. The figures and tables in Sections 3.1-3.2 and Figure 1 are included with kind permission from Springer Science+Business Media B.V.

\subsection{Time-integration and Stiffness}

The two different formulations (the standard and the characteristic) of the boundary conditions were tested. The linear friction law was used in Figure 2 for both cases. Figure 2 show that the spectral radius of non-characteristic formulation increased
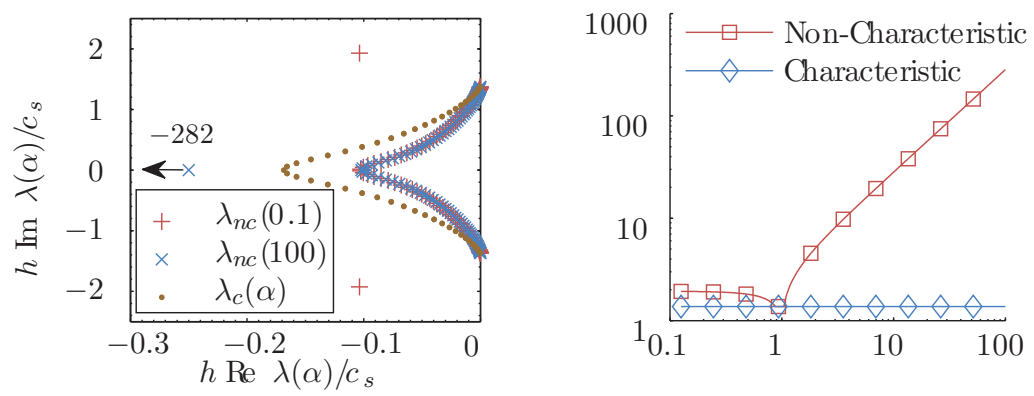

Fig. 2 The spectrum (left figure) using the standard (non-characteristic) boundary conditions and the spectral radius of the two formulations (to the right).

linearly with $\alpha$ and that the characteristic formulation was independent of $\alpha$. The characteristic boundary conditions were clearly less stiff and will be used in the reminder of the paper.

\subsection{Accuracy}

The formulation is tested using the friction law $F(V)=100 \operatorname{arcsinh}(100 \mathrm{~V})$. The initial condition is $\mathbf{q}_{i}(0)=1000 \mathrm{~g}\left(y_{i}\right)(1,1)^{T}$ where

$$
g(y)=\sin (20 \pi y) \exp \left(-\frac{1}{2}\left(y-\frac{1}{2}\right)^{2}\right),
$$

which corresponds to a wave packet moving to the left (into the fault). We compare weak and strong (injection type) of boundary conditions. Strong implementation 
of boundary conditions mean that the solution values at boundaries are identical (overwritten) to the boundary data. Note, there is no stability proof for the strong implementation.

\subsubsection{Accuracy for Short Times}

Time is truncated at $t_{e n d}=1$ and the domain at $y^{*}=1$ with a homogeneous nonreflecting boundary condition.

\begin{tabular}{rrrrrrrr}
\multicolumn{4}{c}{ Injection Method SAT Method } \\
\multicolumn{4}{c}{ Rate Estimate } \\
\multicolumn{3}{c}{$\begin{array}{c}\text { Rate Estimate } \\
\mathrm{N}\end{array} 2^{\text {nd }}$} & $3^{\text {rd }}$ & $4^{\text {th }}$ & $2^{\text {nd }}$ & $3^{\text {rd }}$ & $4^{\text {th }}$ \\
40 & 0.5 & 1.0 & 1.5 & 0.4 & 1.0 & 1.8 \\
80 & 0.7 & 2.1 & 1.7 & 0.8 & 2.0 & 1.3 \\
160 & 1.7 & 1.6 & 2.8 & 1.8 & 1.8 & 3.3 \\
320 & 1.0 & 2.7 & 2.5 & 1.0 & 2.6 & 2.1 \\
640 & 2.1 & 2.7 & 3.3 & 2.2 & 2.8 & 3.5 \\
1280 & 2.1 & 3.3 & 4.1 & 2.1 & 3.3 & 4.4 \\
2560 & 2.2 & 4.1 & 4.8 & 2.2 & 4.2 & 5.1 \\
5120 & 2.4 & 4.4 & 4.7 & 2.4 & 4.5 & 4.9
\end{tabular}

The strong and weak formulations gave approximately the same convergence rates.

\subsubsection{Accuracy for Long Times}

Now, time is truncated at $t_{e n d}=200$ and the domain again at $y^{*}=1$ with a completely reflecting boundary condition. As can be seen in Figure 3, the strong bound-
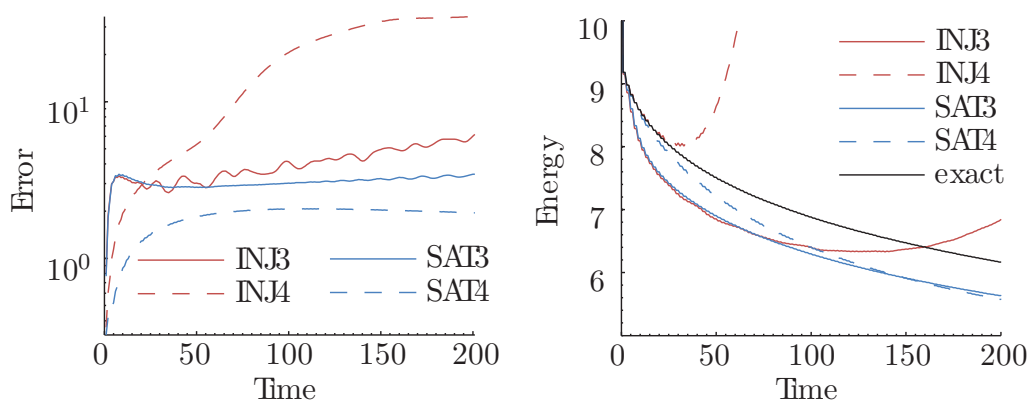

Fig. 3 The long time error growth (left figure) and energy decay (right figure) using the strong and weak implementation of the characteristic boundary formulation. 
ary condition lead to long time error and energy growth while the weak boundary condition had an error bound and the energy decay were close to the exact one.

\subsubsection{The Necessity of Error Bounds for Long Time Calculations}

Here we again truncated at time at $t_{\text {end }}=200$ and the domain at $y^{*}=1$ with a completely reflecting boundary condition. As can be seen in Figure 4, the strong
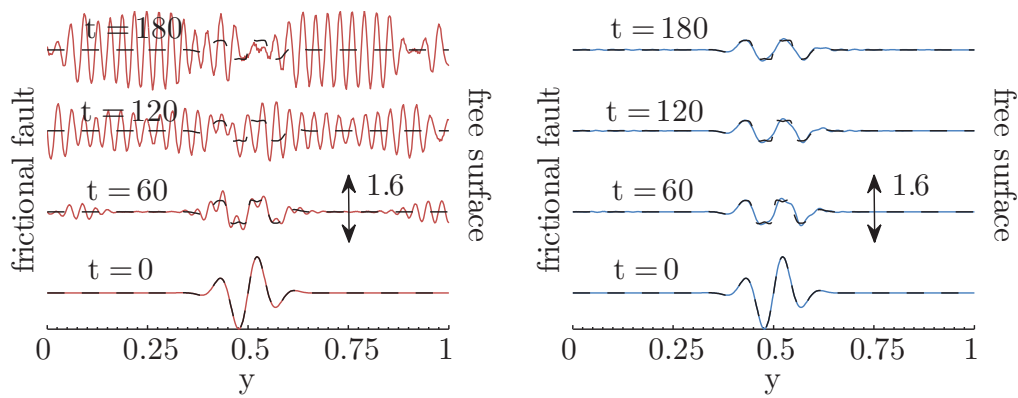

Fig. 4 The long time error growth (left figure) and energy decay (right figure) using the strong and weak implementation of the characteristic boundary formulation.

boundary condition lead to a completely contaminated solution for long times. The weak boundary condition lead to a solution with accurate information even after long times.

\subsection{Application to a Subduction Zone Megathrust Earthquake}

We now consider a more complex application problem to demonstrate the full potential of the method. Note that the full methodology is presented in [8],[9],[7],[4]. The problem is motivated by the recent magnitude 9.0 Tohoku-Oki, Japan, megathrust earthquake and the resulting tsunami. The specific geometry we consider is shown in Figure 5, and is loosely based on the subduction zone structure in the vicinity of the Japan trench.

The Pacific Plate is being sub-ducted to the west beneath the North American / Okhotsk Plate, with relative motion across the plate interface (the fault) occurring during megathrust earthquakes. The Japanese island of Honshu lies at the left edge of the domain, and the upper boundary of the entire computational domain is the seaoor (with the ocean deepening oshore until it reaches a maximum depth of about $7 \mathrm{~km}$ at the trench). Slip along the plate interface causes vertical deformation of 


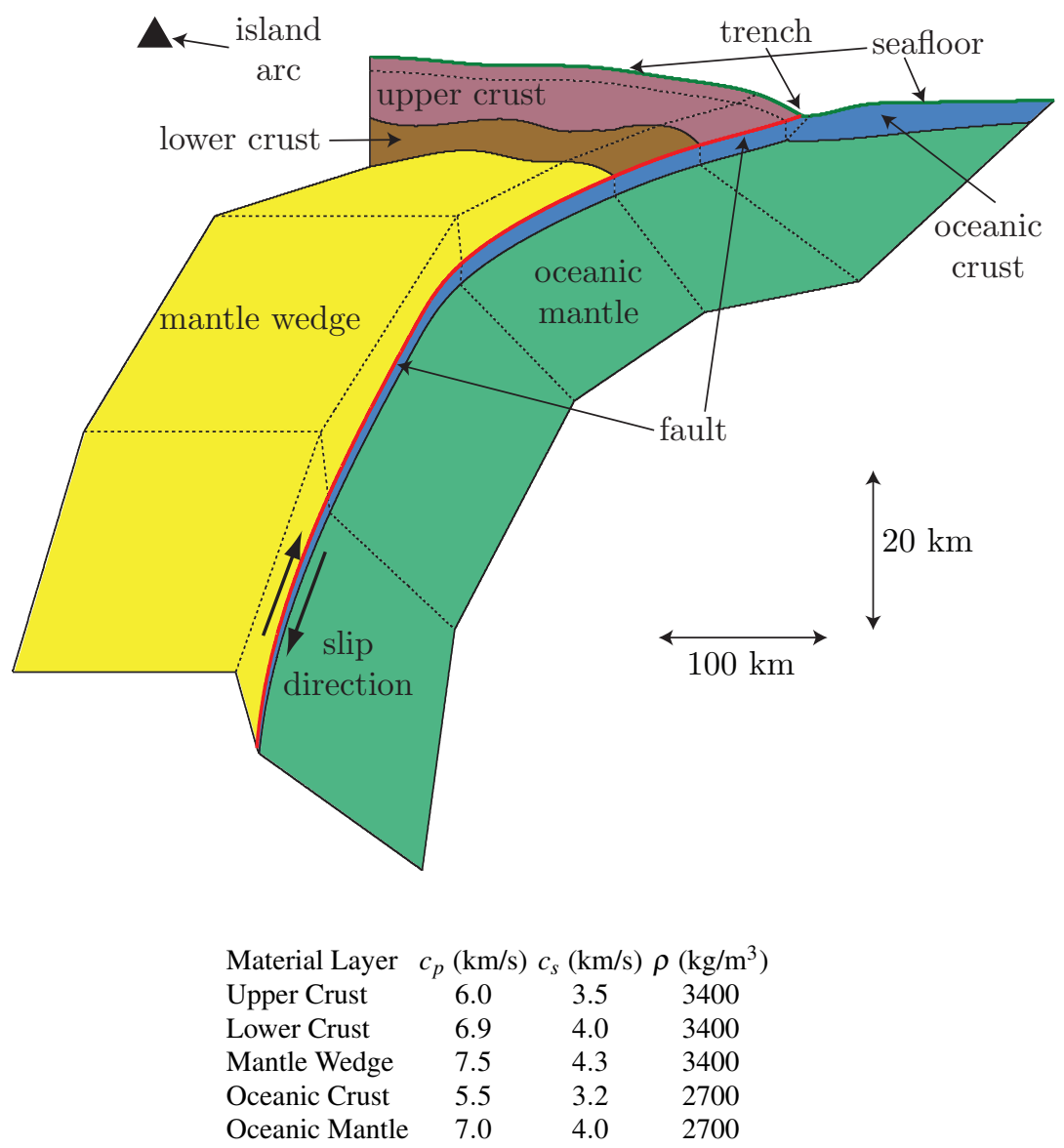

Fig. 5 Subduction zone geometry for megathrust earthquake problem, shown with a factor of 5.25 vertical exaggeration. Different materials are signified with different colors. Solid lines indicate boundaries between the different materials and dotted lines indicate the computational (multi-block grid) boundaries. The fault is highlighted with a thicker solid red line and the seafloor (traction-free surface) is represented with a solid green line. All other boundaries are absorbing boundaries. The accompanying table lists material properties.

the seaoor, causing uplift or subsidence of the overlying water layer. Gravity waves (tsunamis) occur as the sea surface returns to an equilibrium level.

The east (Pacific Plate) side is idealized with a two-layer model (oceanic crust and mantle). As the Pacific Plate dives beneath the North American / Okhotsk Plate, it crosses several material layers (idealized here as upper and lower crust and the mantle wedge). We do not include an ocean layer in this model, due to the large impedance contrast between water and rock, and instead approximate the seaoor as a traction-free surface. The small angle between the seafloor and the fault (about $5^{\circ}$ ) creates an extremely challenging geometry. 
(a) horizontal particle velocity $(\mathrm{m} / \mathrm{s})$ at $\mathrm{t}=15 \mathrm{~s}$

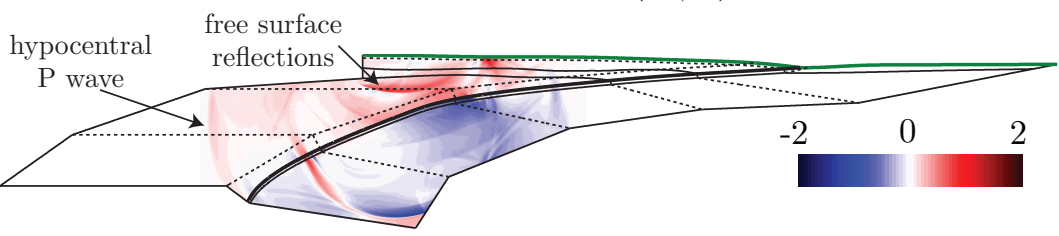

vertical particle velocity $(\mathrm{m} / \mathrm{s})$ at $\mathrm{t}=15 \mathrm{~s}$

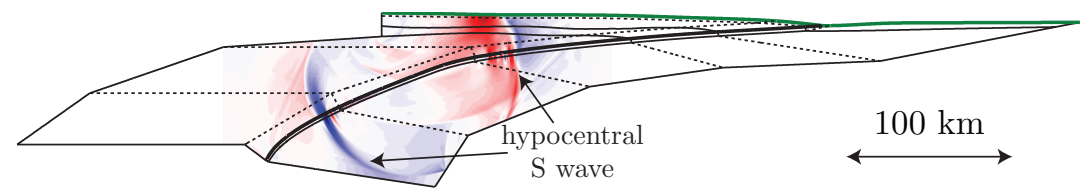

(b)

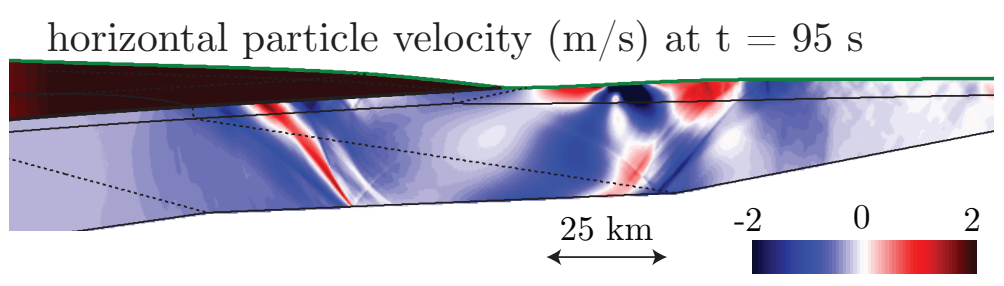

vertical particle velocity $(\mathrm{m} / \mathrm{s})$ at $\mathrm{t}=95 \mathrm{~s}$

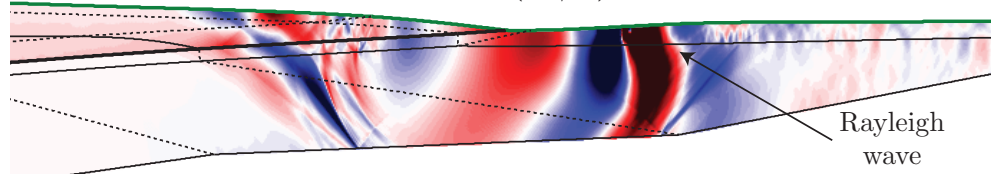

Fig. 6 (a) Wave field at $t=15 \mathrm{~s}$ for the subduction zone megathrust earthquake. The actively slipping part of the fault lies slightly behind the hypo-central S-wave front. (b) Wave field at $t=95$ $\mathrm{s}$ shortly after the rupture has reached the trench. Figures are to scale with no vertical exaggeration. Note different scale bars in (a) and (b).

For this problem, the method must be extended to handle multi-block interfaces, far-field boundaries, complex initial conditions, realistic friction laws and a multitude of other complex physical considerations. We do not intend to go through this in this paper, but refer the reader to [9],[7],[4].

We conduct the simulation at two levels of resolution. The low resolution run has $\sim 5 \times 10^{6}$ grid points (2123 in the $\xi$-direction and 2316 in the $\eta$-direction) with a minimum grid spacing along the fault, interfaces, and exterior boundaries of $100 \mathrm{~m}$, and interior minimum and maximum grid spacings $h_{\min }=0.19 \mathrm{~m}$ and $h_{\max }=200 \mathrm{~m}$, respectively, where $h_{\min }$ is defined and $h_{\max }$ is defined similarly. The time step is $\Delta t=1.5625 \times 10^{-4} \mathrm{~s}$, corresponding to an $\mathrm{S}$-wave CFL of 0.35 . The $200 \mathrm{~s}$ simulation requires $1.28 \times 10^{6}$ time steps. The high resolution run has twice the grid resolution $\left(\sim 10^{7}\right.$ grid points and $2.56 \times 10^{6}$ time steps $)$. 
6(a) shows the wave-field at $15 \mathrm{~s}$. The relative motion of the North American / Okhotsk Plate and the Pacific Plate is consistent with the sense of slip indicated in Figure 5. For the Japan trench, the island arc would be on the North American / Okhotsk Plate side, approximately $250 \mathrm{~km}$ from the trench axis (the intersection of the fault with the seafloor). 6 (b) shows the wave field at $95 \mathrm{~s}$, shortly after the rupture has reached the trench. The material on the North American / Okhotsk Plate side is moving rapidly to the right, due to an extreme reduction in normal stress (and thus fault strength) from wave reflections off the seafloor. The wave field is quite rich in structure, and includes dispersed Rayleigh waves propagating along the seafloor in the oceanic layers. These details are lost in more simplified models that simplify the physics and geometric complexity.

\section{Conclusions}

Linear and nonlinear boundary conditions for wave propagation problems have been considered. The concepts of well-posedness and stability were discussed by focusing on a specific example of boundary treatment occurring in the modeling of earthquakes.

The boundary condition were formulated in a linear and nonlinear way and implemented in a characteristic and non-characteristic way. These differences were discussed and the implications and difficulties were pointed out.

To discretize in space we used summation-by-parts difference operators and imposed the boundary conditions weakly using the Simultaneous Approximation Term (SAT) technique.

It was found that the same conditions that lead to well-posedness for the noncharacteristic boundary conditions also lead to stability and convergence of the numerical solution. This goes for both the linear and nonlinear boundary condition, even though the nonlinear case was more complex.

The conditions that lead to well-posedness for the characteristic boundary conditions were different than the ones required for the non-characteristic conditions, except in the linear case. The conditions in the nonlinear case were less restrictive.

The conditions that lead to well-posedness for characteristic boundary conditions also lead to stability for the discrete problem, but not to convergence. To guarantee convergence we needed to sharpen the conditions to the same level as in the noncharacteristic case for the nonlinear case.

Both types of boundary conditions lead to error bounded schemes if implemented weakly.

Numerical simulations that illustrate the theoretical discussion are presented. We show that the correct accuracy as well as stability properties both for short and long times agree with the theoretical predictions. Finally, an application that show that the methodology can be used for practical problems is discussed. 


\section{References}

1. S. Abarbanel, A. Ditkowski, and B. Gustafsson. On error bounds of finite difference approximations to partial differential equations - temporal behavior and rate of convergence. Journal of Scientific Computing, 15(1):79-116, 2000.

2. J. Berg and J. Nordström. Stable Robin solid wall boundary conditions for the Navier-Stokes equations. Journal of Computational Physics, 230:7519-7532, 2011.

3. M.H. Carpenter, J. Nordström, and D. Gottlieb. A stable and conservative interface treatment of arbitrary spatial accuracy. Journal of Computational Physics, 148:341-365, 1999.

4. E. M. Dunham and J. E. Kozdon. Rupture dynamics of subduction megathrust earthquakes. In Abstract T31E-05 presented at 2011 Fall Meeting, San Francisco, California, USA, 2011.

5. J. Gong and J. Nordström. Interface procedures for finite difference approximations of the advectiondiffusion equation. Journal of Computational and Applied Mathematics, 236(5):602620, 2011.

6. B. Gustafsson, H.-O. Kreiss, and J. Oliger. Time Dependent Problems and Difference Methods. John Wiley \& Sons, Inc., 1995.

7. J. E. Kozdon and E. M. Dunham. Rupture to the trench in dynamic models of the tohoku-oki earthquake. In Abstract U51B-0041 presented at 2011 Fall Meeting, AGU, San Francisco, California, USA, 2011.

8. J. E. Kozdon, E. M. Dunham, and J. Nordström. Interaction of waves with frictional interfaces using summation-by-parts difference operators: Weak enforcement of nonlinear boundary conditions. Journal of Scientific Computing, 50(2):341-367, 2012.

9. J. E. Kozdon, Eric M. Dunham, and J. Nordström. Simulation of dynamic earthquake ruptures in complex geometries using high-order finite difference methods. Technical Report 2012:2, Linköping University, Computational Mathematics, 2012.

10. H.-O. Kreiss and G. Scherer. Finite element and finite difference methods for hyperbolic partial differential equations, in: C. De Boor (Ed.), Mathematical Aspects of Finite Elements in Partial Differential Equation. Academic Press, New York, 1974.

11. K. Mattsson and J. Nordström. Summation by parts operators for finite difference approximations of second derivatives. Journal of Computational Physics, 199:503-540, 2004.

12. J. Nordström. Error bounded schemes for time-dependent hyperbolic problems. SIAM J. Sci. Comp., 30(1):46-59, 2007.

13. J. Nordström and M. H. Carpenter. Boundary and interface conditions for high order finite difference methods applied to the Euler and Navier-Stokes equations. Journal of Computational Physics, 148:621-645, 1999.

14. J. Nordström and M. H. Carpenter. High-order finite difference methods, multidimensional linear problems and curvilinear coordinates. Journal of Computational Physics, 173:149-174, 2001.

15. J. Nordström, J. Gong, E. van der Weide, and M. Svärd. A stable and conservative high order multi-block method for the compressible Navier-Stokes equations. Journal of Computational Physics, 228(24):9020-9035, 2009.

16. J. Nordström and M. Svärd. Well-posed boundary conditions for the Navier-Stokes equations. SIAM Journal on Numerical Analysis, 43(3):1231-1255, 2005.

17. Jan Nordström. Conservative finite difference formulations, variable coefficients, energy estimates and artificial dissipation. Journal of Scientific Computing, 29:375-404, 2006.

18. B. Strand. Summation by parts for finite difference approximation for d/dx. Journal of Computational Physics, 110(1):47-67, 1994

19. M. Svärd, M.H. Carpenter, and J. Nordström. A stable high-order finite difference scheme for the compressible Navier-Stokes equations: far-field boundary conditions. Journal of Computational Physics, 225(1):1020-1038, 2007.

20. M. Svärd and J. Nordström. On the order of accuracy for difference approximations of initialboundary value problems. Journal of Computational Physics, 218(1):333-352, 2006.

21. M. Svärd and J. Nordström. A stable high-order finite difference scheme for the compressible Navier-Stokes equations: No-slip wall boundary conditions. Journal of Computational Physics, 227(10):4805-4824, 2008. 